\title{
Alternative psychosis (forced normalisation) in epilepsy
}

V T R Ntsanwisi, MB ChB, MMed (Psych)

S T Rataemane, MB ChB, FF Psych (SA), Child and Adolescent Psychiatry (UK)

Department of Psychiatry, University of Limpopo (MEDUNSA campus), PO Medunsa, 0204

D S Magazi, MB ChB, MMed, FCP (Neurol) SA Department of Neurology, University of Limpopo (MEDUNSA campus)

Forced normalisation is a paradoxical relationship between seizure activity and behavioural problems. A 20-year-old man with recurrent refractory tonic-clonic epilepsy experienced forced normalisation while on medication with multiple anti- epileptic drugs (valproate sodium, carbamazepine and topiramate). A reduction in the seizure burden correlated with sudden behavioural changes manifesting with aggressive outbursts and violence.

The case may help clarify the mechanism of forced normalisation while providing some helpful hints regarding the diagnosis and treatment of symptoms observed in recurrent refractory seizures.

Brief episodes of abnormal behaviour have been recorded after dramatic reduction of seizures using anti-epileptic drugs (AEDs). This phenomenon is called alternative psychosis, or forced normalisation when supportive electro-encephalogram (EEG) evidence is available.

Landolt was the first to report improvement in EEG activity during periods of abnormal behaviour. ${ }^{.9}$ The mechanism of forced normalisation is still not fully understood, although the kindling phenomenon, the phenomenon of long-term potentiation and the channel disorder paradigm have all been proposed as possible explanations. ${ }^{10.11}$ Another theory relating to this phenomenon states that epileptiform discharges may mimic electroconvulsive therapy in a focal area and this seizure suppression may lead to psychopathology. ${ }^{2}$

Patients with refractory temporal lobe epilepsy who undergo unilateral anterior temporal lobectomy have been observed to develop a de novo psychosis with diminished seizures.
This is thought to be an alternative psychosis related to forced normalisation of the EEG. . $^{81214}$

The absence of clear diagnostic criteria for forced normalisation has been an impediment both to its routine diagnosis and to further research. Research into this phenomenon has been confined mainly to case reports (as in this case) or to retrospective studies.

Anticonvulsant drugs have been associated with new-onset depression and psychosis. If anticonvulsants have recently been changed, this should always be considered as a potential cause of a new or worsening depressive or psychotic illness. ${ }^{15,16}$

There are case reports in the literature describing psychosis relating to topiramate, zonisamide and levetracetam (see Table I for a list of drugs implicated in forced normalisation). Some of these reports may relate to the process of forced normalisation, in which a diminished frequency of seizures allows psychotic symptoms to emerge. ${ }^{15}$

Krishnamoorthy and Trimble list questions posed by other researchers that still remain unanswered: ${ }^{\circ}$

1. Should the EEG necessarily be completely normal for this diagnosis to be made, or should relative normalisation also be included?

2. What is the relationship of the EEG to suppression of seizures?

3. Is alternative psychosis the expression of forced normalisation, a variant, or unrelated to it?

The current approach is wider inclusion of cases that show a decrease in seizure frequency with both relative and complete normalisation of the EEG.

The following are the proposed criteria for forced normalisation: ${ }^{\circ}$

Primary (essential) criteria:

1. Established diagnosis of epilepsy based on clinical history, EEG and imaging

2. Presence of a behavioural disturbance of acute/sub-acute onset characterised by one of the following

- psychosis with thought disorder, delusions, hallucinations

- significant mood change, mania/hypomania or depression

- anxiety with depersonalisation, derealisation

- hysteria: motor, sensory, abasia 
3A. Reduction in the total number of spikes counted in a 60-minute awake EEG recording with a normal 16-channel machine, using standard 10 - 20 electrode placement, by over $50 \%$ compared with a similar recording performed during a normal state of behaviour

or

3B. Report of complete cessation of seizures for at least 1 week, corroborated by a relative or a carer.

Supportive criteria:

1. Recent change (within 30 days) of pharmaco-therapeutic regimen

2. Report of similar episodes of seizure cessation and behavioural disturbance in the past, from close relative, carer or general practitioner, or documentation of these in hospital records, with or without EEG evidence. This may or may not be linked with anticonvulsant drugs.

The diagnosis is made in the presence of:

Primary criteria 1, 2 and $3 \mathrm{~A}$

or

Primary criteria 1, 2 and $3 \mathrm{~B}$ and one supportive criterion. ${ }^{18}$

Trimble makes no specific recommendation regarding the type of imaging of epilepsy; however, according to Kanner et al., the presence of interictal psychosis should prompt the clinician to order high-resolution magnetic resonance imaging (MRI) in search of small tumours (harmatomas and ganglionomas), some

\begin{tabular}{l} 
Table I. Drugs implicated in forced normalisation \\
\hline Ethosuximide $^{18}$ \\
Lamotrigine $^{26}$ \\
Vigabatrin $^{26}$ \\
Levetiracetam $^{28}$ \\
Topiramate $^{29}$ \\
\end{tabular}

of which may not be detectable on the standard MRI and may cause refractory epilepsy. ${ }^{19}$ Fluoro-deoxyglucose-positron emission tomography (FDG-PET) may show hypometabolic areas that correlate with epileptic foci as revealed by the EEG. ${ }^{19}$ However, the challenge in the South African context is limited availability of some of these diagnostic tools.

\section{Case report}

A 20-year-old man with recurrent refractory tonic-clonic epilepsy was referred to the liaison psychiatry registrar at George Mukhari Hospital by the neurology registrar after he had an altercation with the nursing staff in the neurology ward.

The patient had been treated for recurrent refractory epilepsy by the neurology department since 1996. He was on multiple AEDs. His current drug regimen was sodium valproate 1000 $\mathrm{mg}$ in the morning and $1500 \mathrm{mg}$ at night, carbamazepine 400 mg twice a day and topiramate $50 \mathrm{mg}$ twice a day. There had been a substantial reduction in his seizure burden from a seizure approximately every 2 days a year ago before the introduction of the current drug regimen to 3 - 4 seizures a month after its introduction. The change in behaviour had been observed for the first time by the family in the 6 months following the reduction in seizure frequency.

For the first time, the patient had presented with bouts of verbal and physical aggression and even violence. He had recently assaulted his grandmother and also chased his elder brother with a knife with the intention of harming him. According to the family there had been no provocation prior to the incident, and the patient subsequently apologised, showing remorse upon realisation of the wrongfulness of his actions. The patient admitted to taking alcohol occasionally at social functions. He drank only beer, three or four $350 \mathrm{ml}$ cans at a sitting. He denied smoking cannabis or taking any other illicit substance. There was a positive family history of epilepsy, his deceased maternal aunt having been affected.

According to his mother, the patient had been delivered vaginally at term, although the labour had been prolonged and he had

\section{Table II. A list of published case reports and case series on forced normalisation/alternative psychosis}

$\begin{array}{llll}\text { Author } & \text { Year } & \text { Country } & \text { No. of patients } \\ \text { Matsuura' }^{12} & 1997 & \text { Japan } & \text { Meta-analysis 14 cases } \\ \text { Mula and Monaco }^{14} & 2003 & \text { Italy } & 103 \\ \text { Bootsma et al." }^{11} & 2004 & \text { Netherlands } & 470 \\ \text { Chkhenkeli and Geladze }^{7} & 2005 & \text { Georgia } & 150 \\ \text { Ohara et al. }^{10} & 2006 & \text { Japan } & \text { Single case report } \\ \text { Trimble and Rusch } & 2007 & \text { UK } & 89\end{array}$


a 2-week stay in the neonatal ward due to severe neonatal jaundice. The developmental milestones were reported to be normal. According to the mother the patient had passed every class at the first attempt up to Grade 5 in 1999, but was forced to stop school because of his severe epilepsy. However, no school report was available to substantiate her account of his academic performance. His premorbid personality was described as quiet and soft-spoken. According to his mother, the patient had undergone a personality change in the past 12 months, having become impulsive, aggressive and violent. He became prone to temper tantrums, banging doors, and would throw himself to the floor if he did not get his way.

The findings on physical examination were normal, with a blood pressure of $130 / 90 \mathrm{mmHg}$, a regular pulse rate of 72 beats/min, and no evidence of lymphadenopathy or thyroid enlargement. The cardiovascular, respiratory, musculoskeletal and abdominal systems appeared normal. The pupils were equal and reactive to light. The optic disc appeared normal on fundoscopy. The cranial nerves were intact and examination of the upper and lower limbs revealed normal power, tone, reflexes, co-ordination and sensation.

On mental status examination, the patient's behaviour was appropriate, though conflict was observed in his interaction with his mother, leading to irritable mood. Affect was appropriate, and there was mild psychomotor retardation. His speech was slow in rhythm but normal in quantity and volume. He was orientated to person, place and time. Attention and concentration were poor; he was able to do the first two of the serial 7 subtraction 193, 86), but there was latency of response, pointing towards slowing of cognitive speed. Memory was intact, and there were no perceptual distortions.

The patient's thinking process was logical and goal directed, and the content showed no psychopathology. Insight was partial and judgement was fair, as evidenced by the retaliation after provocation, and then showing remorse. Abstract thinking was normal. His intelligence was borderline towards sub-average. He had a Mini-Mental State score of 23/30, pointing towards cognitive deficit.

Since there was no aura, the patient was clinically classified as having primary generalised epilepsy. The EEG showed multifocal spike and wave abnormalities.

This patient's clinical course meets the criteria for alternative psychosis and forced normalisation of the EEG. We accommodate the possibility of coincidence, in other words no causal link between seizure frequency and the emergence of aggressive behaviour. In this case, however, we are persuaded towards there being a causal link in that this was a new development that emerged in the 12th year only after a change in drug regimen that resulted in a drastic reduction in seizure frequency. The patient satisfies Krishnamoorthy and Trimble's criteria 1, 2, 3B and one supportive criterion.

A differential diagnosis of episodic dyscontrol syndrome was made; this is characterised by sudden episodes of spontaneously released violence with minimal provocation that terminate abruptly. These episodes are precipitated by small amounts of alcohol and anticonvulsant drugs, especially the benzodiazepines, and are considered to be a form of paradoxical reaction that occurs in individuals with pre-existing brain damage, such as represented by this patient, who sustained neuronal injury as a result of recurrent refractory seizures. ${ }^{20}$

Psychosis secondary to epilepsy is an important consideration in this case scenario. Patients with chronic epilepsy lespecially those with temporal lobe epilepsy) eventually succumb to a schizophrenia-like state. ${ }^{2 !}$

\section{Discussion}

The patient had been suffering from recurrent epilepsy since 1996, when he was 8 years old. His medical records show that he was initially treated with phenytoin, which was stopped due to toxicity and gingival hypertrophy.

Different regimens were tried without much success. Recently his seizure burden reduced to 3 - 4 seizures a month from a seizure every 2 days, but then episodes of abnormal behaviour characterised by aggressive outbursts and impulsivity came to the fore for the first time (alternative psychosis or forced normalisation). The new adjunct AEDs have been observed to be associated with this phenomenon. Trimble and Schmitz ${ }^{22}$ have warned clinicians about these side-effects and have suggested slower titration of medication for seizure control as a measure to prevent them.

Initial drug therapy to control seizure activity is based on the specific type of seizure. Monotherapy is initiated with a single agent until seizure remission or signs of toxicity. Patients on monotherapy fare much better than those on combination therapy regarding compliance and side-effects. In the event of failure to control seizures with the first drug, monotherapy with a different anti-epileptic is suggested before resorting to augmentation strategies with different AEDs. ${ }^{23,24}$

Most AEDs exert their effect by inhibiting generation of an electric discharge from a focal area or by blocking the propagation of an abnormal electric charge to an adjacent brain area. They accomplish this by a variety of mechanisms, including:

- inhibition of voltage-gated channels $(\mathrm{Na}+, \mathrm{K}+$ or $\mathrm{Ca} 2+$ )

- enhancement of inhibitory GABA-ergic impulses

- attenuation of excitatory glutamate transmission. ${ }^{23,2527}$ 
Systemic data on the psychiatric adverse effects of primary AEDs are very limited and mainly based on empirical data and small case series (Table II).

Drugs with a GABA-ergic mechanism of action have sedating, anxiolytic and anti-manic properties, while drugs with a glutametergic mechanism of action have activating, anxiogenic and antidepressant effects. Tiagabine and vigabatrin are examples of the first category, and lamotrigine an example of the second.

Topiramate was the second-line augmenting drug used in the present case. Its manner of action is multimodal and involves blockage of voltage-gated sodium channels, potentiation of GABA-ergic transmission and inhibition of excitatory glutametergic pathways through an action at the AMPA receptor site. ${ }^{28}$

Neuropsychiatric manifestations in the form of psychosis, affective disorders and behavioural disorders have emerged in patients on topiramate, vigabatrin and tiagabine. Trimble and Rusch $^{29}$ carried out a retrospective case study on 89 patients who developed behavioural symptoms during treatment with topiramate, vigabatrin and tiagabine. It was found that $99 \%$ of the patients suffered from complex partial seizures with or without secondary generalisation. More than half were on polypharmacy with two or more AEDs. Nearly two-thirds had a previous psychiatric history.

A strong association was found between the type of previous psychiatric illness and the type of emerging psychiatric problem. A seizure-free period was observed in more than half of the patients before they developed the psychiatric symptoms, ${ }^{29}$ further entrenching the perceived paradoxical relationship between reduction in seizure frequency and emergence of psychiatric symptoms (i.e. forced normalisation/alternative psychosis).

\section{Conclusion}

Although there was a substantial reduction in seizure burden in this patient on medication with multiple AEDs, behavioural changes manifesting with aggressive outbursts and violence appeared for the first time.

The goal in the treatment of epilepsy is to render the patient seizure-free, or at least to bring about a significant decrease in seizure frequency. Clinicians need to realise that seizure control in a sub-group of these patients may lead to alternative forms of neuropsychiatric dysfunction such as forced normalisation. ${ }^{30}$

The prevalence of this phenomenon could potentially grow substantially with the wider availability and use of the adjunct antiepileptic drugs, as observed by Trimble. ${ }^{31}$

Finally, we suggest a change in the nomenclature, as the current terms 'forced normalisation' and 'alternative psychosis' do not adequately describe the clinical cause and outcome of this condition. We propose the new name of 'ictal suppression disorder', which aptly describes this phenomenon and removes the ambiguity created by the current terms.

\section{References}

1. Cummings JL, Trimble MR. Concise Guide to Neuropsychiatry and Behavioral Neurology. 2nd ed. Washington, DC: American Psychiatric Publishing Inc., 2002:139-147.

2. Nadkarni S, Devinsky O. Psychotropic effects of antiepileptic drugs. Epilepsy Curr 2005;5:176-181

3. Pakalnis A, Drake ME, Kuruvilla J, et al. Forced normalization. Arch Neurol 1987;44:289-292

4. Schmitz B. Effects of antiepileptic drugs on mood and behaviour. Epilepsia 2006; 47:28-33.

5. Nadkami S, Aredo V, Devinsky O. Psychosis in epilepsy patients. Epilepsia 2007;48:17-19.

6. Krishnamoorthy ES, Trimble MR. Forced normalization: clinical and therapeutic relevance. Epilepsia 1999:40:57-64.

7. Chkhenkeli SA, Geladze TS. 'Forced normalization' of the EEG and some mechanisms of psychopathological manifestations in epileptics. Neurosci Behav Physiol 1982;12:113-117.

8. Torta R, Keller R. Behavioural psychotic and anxiety disorders in epilepsy, etiology, clinical features and therapeutic implications. Epilepsia 1999;40:2-20.

9. Semple D, Smyth R, Burn J, et al. Oxford Handbook of Psychiatry. Oxford: Oxford University Press, 2005: 159.

10. Ohara T, Monji A, Onitsuka T, et al. Interictal psychosis after stroke with forced normalization. J Neuropsychiatry Clin Neurosci 2006; 1 8:557-560.

11. Bootsma HP, Coolen F, Aldenkamp AP, et al. Topiramate in clinical practice: Long term experience in patients with refractory epilepsy referred to a tertiary epilepsy centre. Epilepsy Behav 2004;5:380-387.

12. Matsuura M. Psychosis of epilepsy with special reference to anterior lobectomy Epilepsia 1997; 38:32-34.

13. Kohler CG, Carran MA, Bilker W, et al. Association of fear auras with mood and anxiety disorders after temporal lobectomy. Epilepsia 2001;42:674-68 1.

14. Mula M, Monaco F. Antiepileptic drugs and psychopathology of epilepsy: An update. Epileptic Disord 2009; 1 1:1-9

15. Taylor D, Paton C, Kerwin R. The Maudsley Prescribing Guidelines. 9th ed. London: Informa Health Care, 2007:353-363.

16. Mula M, Herdorffer DC, Trimble MR, et al. The role titration schedule of topiramate for the development of depression in patients with epilepsy. Epilepsia 2009; 50: 1072-1076.

17. Tatum WO, French JA, Faught E, et al. Post marketing experience with topiramate and cognition. Epilepsia 2001;42:1 134-1 140

18. Krishnamoorthy ES, Trimble MR, Sander JW, et al. Forced normalization at the interface between epilepsy and psychiatry. Epilepsy Behav 2002;3:303-308.

19. Kanner AM. Psychosis of epilepsy: A neurologist perspective. Epilepsy Behav 2000; 1:219-227

20. Buckley P, Prewette D, Bird J, et al. Examination Notes in Psychiatry. 4th ed. London: Hodder Arnold, 2005:175-184.

21. Glosser G, Zwil A, Glosser D S, et al Psychiatric aspects of temporal lobe epilepsy before and after anterior temporal lobectomy. J Neurol Neurosurg Psychiatry 2000;68:53-58.

22. Trimble MR, Schmitz B, eds. Forced Normalisation and Alternative Psychoses of Epilepsy. Petersfield, Hants: Wrightson Biomedical Publishing, 1998:25-48

23. Harvey RA, Champe PC, Howland RD, et al. Pharmacology. 3rd ed. Philadelphia: Lippincott, 2006:169-180.

24. Stahl SM. Essential Pharmacology, the Prescriber's Guide. New York: Cambridge University Press, 2006:499-504.

25. Katzung BG. Basic and Clinical Pharmacology. 10th ed. New York: McGraw Hill, 2007:374-394

26. McElroy SL, Keck PE Jr, Post RM. Antiepileptic drugs to treat psychiatric disorders. Medical Psychiatry Series 39. New York: Informa Healthcare, 2008: 17-32.

27. Karlovasitou A, Stergiou B. Effectiveness and neuropsychiatric side effects of anticonvulsant drugs in psychiatric disorders. Encephalos 46;1:35-39.

28. Perucca E. A pharmacological and clinical review on topiramate, a new antiepileptic drug. Pharmacol Res 1997:35:241-256.

29. Trimble MR, Rusch N. Psychiatric symptoms after therapy with new antiepileptic drugs, psychopathological and seizure related variables. Seizure 2008;9:249-254.

30. Mula M Trimble MR. The importance of being seizure free: Topiramate and psychopathology in epilepsy. Epilepsy Behav 2003:4:430-434

31. Trimble MR. New anti-epileptic drugs and psychopathology. Neuropsychobiology 1998:38:149-151. 